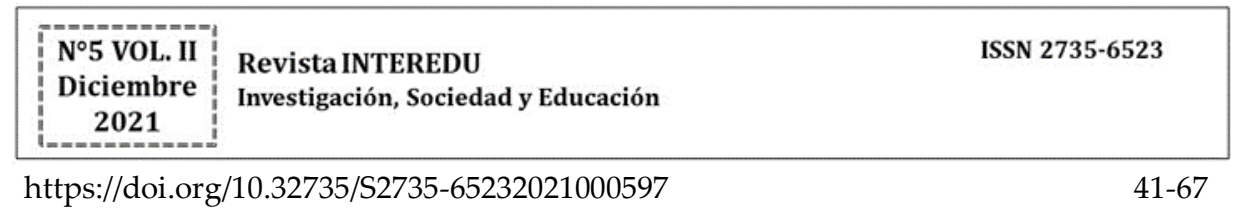

\title{
TRAYECTORIAS ESCOLARES, MIGRACIÓN Y FORMACIÓN DOCENTE: PROCESOS QUE DIALOGAN
}

\author{
School paths, migration and teacher training: processes in dialogue

JULIETA TAQUINI
Universidad Nacional de Tierra del Fuego (Argentina)
jtaquini@untdf.edu.ar
https://orcid.org/0000-0002-9436-8732

\section{RESUMEN}

El presente trabajo de investigación indaga en las trayectorias escolares de mujeres de origen boliviano que actualmente residen en la ciudad argentina de Ushuaia, en Tierra del Fuego; y que accedieron a la formación docente en esa ciudad. Se propuso una investigación desde una perspectiva socioantropológica (Achilli, 2005) con un diseño flexible que nos permitió detallar cómo se configuran y cómo se manifiestan los fenómenos inscriptos en matrices socioculturales amplias. A partir de esta investigación podemos decir que las experiencias vividas por estas mujeres en los procesos migratorios atravesados, en diálogo con la formación docente, favorecieron la construcción de nuevos sentidos respecto de las condiciones materiales y simbólicas de sus trayectorias escolares, como así también entrever perspectivas para transformarlas.

Palabras clave: Antropología de la educación; desigualdad social; formación de docentes; migración; mujeres estudiantes.

1 Licenciada en Ciencias Sociales y Humanidades (UNQ), DSP en Migraciones, Movilidades e Interculturalidad en América Latina (FLACSO). Maestranda en Ciencias Sociales y Humanidades (UNQ). 


\section{Julieta Taquini}

\section{ABSTRACT}

This research paper studies the school trajectories of Bolivian women who are currently residing in Ushuaia, Argentina, in Tierra del Fuego, and had been part of the local teacher-training process, in that city. An investigation was proposed from a socio-anthropological perspective (Achilli, 2005) with a flexible design which have allowed us to provide an in-detail description of how such phenomena, inscribed in broad sociocultural matrices, are both configured and manifested. From this research, we can say that the experiences lived by these migrant women, who have also faced a pedagogy-training process, favored the construction of new meanings regarding the material and symbolic conditions of their school trajectories, enabling new settings to transform them.

Key words: Educational anthropology; social inequality; teacher education; migration; women students.

\section{INTRODUCCIÓN}

En el presente artículo se presentan algunos hallazgos, resultado del Trabajo Final de la Maestría en Ciencias Sociales y Humanidades de la Universidad Nacional de Quilmes (Argentina) intitulada, Migración, género y formación docente: trayectorias escolares de mujeres de origen boliviano de la ciudad de Ushuaia. Su objetivo fue indagar en las trayectorias escolares atravesadas por procesos migratorios de mujeres de origen boliviano que residen en la ciudad de Ushuaia y que accedieron, transitaron o finalizaron la formación docente en esa ciudad.

Este trabajo se centra en las particularidades que adquieren dichas trayectorias en el actual contexto socio-histórico y, especialmente, en una ciudad como Ushuaia que posee una singular historia respecto del significado de los movimientos migratorios, dadas las peculiares 42 | INTEREDU № 5 VOL. II (DiCIEMBRE 2021) PÁGs. 41-67. ISSN: 2735-6523 
características de su ubicación geográfica y la configuración histórica de su población (Hermida y Van Aert, 2013).

En este sentido, Mallimaci (2010) nos alerta que en la capital fueguina algunas residencias son más legitimadas que otras; y si bien, según el Instituto de Estadística y Censos de la República Argentina (INDEC), la población migrante de origen boliviano muestra un aumento en el censo de 2010 con respecto a la evidenciada en el censo de $2001^{2}$ (INDEC, 2001, 2010), las representaciones sociales sobre esa comunidad "expresan relaciones discriminatorias, produciendo desigualdad y exclusión” (Mallimaci, 2011, p. 6).

El estudio de la problemática además adquiere relevancia porque, a pesar del creciente aumento de políticas educativas nacionales conocidas como interculturales ${ }^{3}$ que plantean propuestas particularizadas en contextos de presencia indígena o migrante, estas no incluyen a las instituciones de educación superior, en tanto las mismas son reguladas mediante la Ley de Educación Superior №24521 (Congreso de la República Argentina, 1995) que no hace menciones a la noción de interculturalidad (Hecht y Schmidt, 2016).

En este sentido, por un lado, interesa advertir que en Argentina la educación superior universitaria y no universitaria es considerada un

\footnotetext{
${ }^{2}$ La población migrante boliviana ha aumentado en las últimas décadas -de 851 en 2001 a 3376 en 2010 - constituyéndose en la mitad de los extranjeros en el último censo en Ushuaia.

${ }^{3}$ Estas políticas han sido plasmadas en: la Resolución №107 del Consejo Federal de Cultura y Educación (1999), la Resolución №549 del Ministerio de Educación Ciencia y Tecnología de la República Argentina (2004), el Documento del Programa Nacional de Educación Intercultural Bilingüe de la Dirección Nacional de Programas Compensatorios Subsecretaría de Equidad y Calidad Educativa del Ministerio de Educación, Ciencia y Tecnología de la República Argentina (2004), la Ley de Educación Nacional №26.206 (2006), el Documento para la Modalidad de Educación Intercultural Bilingüe en el Sistema Educativo Nacional del Consejo Federal de Educación (2010) y la Resolución №105 del Consejo Federal de Cultura y Educación (2010), entre otros instrumentos legales.
}

INTEREDU № 5 VOL. II (Diciembre 2021) PÁGs. 41-67. ISSN: 2735-6523| 43 


\section{Julieta Taquini}

derecho humano con jerarquía constitucional ${ }^{4}$. Por tanto, el Estado garantiza la gratuidad, el acceso y el desarrollo de las trayectorias educativas de quienes han decidido emprender una carrera en el nivel superior de educación, por medio de un amplio abanico de políticas públicas que ponen en marcha alternativas institucionales, pedagógicas y de promoción de derechos tales como becas y programas de sostén a las trayectorias estudiantiles. Por otro lado, es relevante indicar que las personas migrantes que viven en Argentina tienen el mismo derecho que las nacionales a acceder a la educación en todos los niveles educativos.

Sin embargo, aunque la actual Ley de Migraciones №25871 (Congreso de la República Argentina, 2010) establece que se garantizará el acceso a los distintos niveles educativos con el alcance previsto en la Ley de Educación Nacional № 26206 (Congreso de la República Argentina, 2006) en ese país, según datos arrojados por la Dirección Nacional de Información y Evaluación de la Calidad Educativa (DINIECE, 2010), el porcentaje de inmigrantes extranjeros es muy bajo en todos los niveles de enseñanza del sistema, siendo en el nivel superior no universitario de apenas el 3,2 \% de estudiantes (Dirié y Sosa, 2014), constatándose formas subordinadas de inclusión para este grupo social (Martínez et al., 2011).

Con el fin de abordar las interrogantes planteadas, se decidió un diseño de corte cualitativo en tanto entendemos la realidad como una

\footnotetext{
${ }^{4}$ En el inciso 19 del artículo 75 de la Constitución Nacional de la República Argentina se ordena al Congreso "garanti [zar] los principios de gratuidad y equidad de la educación pública estatal" en todos los niveles educativos. Luego, en el inciso 22 del mismo artículo, se otorga jerarquía constitucional a varios instrumentos internacionales que garantizadores de derechos entre los que se encuentra el Pacto Internacional de Derechos Económicos, Sociales y Culturales (PDESC) (Asamblea general de las Naciones Unidas, 1966) que en su artículo 13 sostiene que la educación superior, en tanto derecho, no puede ver cercenada su gratuidad.

44 | INTEREDU № 5 VOL. II (Diciembre 2021) PÁGS. 41-67. ISSN: 2735-6523
} 
construcción intersubjetiva y múltiple; y a quien investiga y el contexto de interacción, mutuamente influenciados. Se propuso una investigación desde una perspectiva socioantropológica (Achilli, 2005) y un diseño flexible que nos permitió introducir conceptos, categorías y relaciones, así realizar cambios cuando se creyó necesario. Desde este enfoque crítico, la etnografía nos permitió poner en relación las biografías particulares con la historia social e institucional que les toca vivir a los sujetos: es "la posibilidad de recuperar lo particular y significativo desde lo local, pero además de situarlo en una escala social más amplia, en un marco conceptual más general, es la contribución posible de la etnografía en los procesos de transformación educativa" (Rockwell, 2015a, p.34).

En el marco de este estudio, se entenderá la etnografía tanto a la forma de proceder en la investigación de campo como al producto final, en la que la tarea de documentar lo no documentado de la realidad social contribuye en la comprensión de los procesos inscriptos en matrices socioculturales más amplias, recuperando lo particular y lo significativo desde lo local, en tanto síntesis de variados cruces y definiciones contextuales (Rockwell, 2015a).

En cuanto a los instrumentos de construcción de la información, se utilizaron entrevistas en profundidad, ya que al decir de Taylor y Bogdan (1987), están dirigidas "hacia la comprensión de las perspectivas que tienen los informantes respecto de sus vidas, experiencias o situaciones, tal como las expresan con sus propias palabras" (p. 101). También recurrimos al análisis de documentos, como por ejemplo narraciones por parte de las entrevistadas, o las reglamentaciones vigentes en relación al ingreso y permanencia en el nivel educativo en cuestión, además de observaciones a participantes y entrevistas a otros actores significativos de la problemática. 


\section{Julieta Taquini}

El referente empírico que por sus características responde a las necesidades de nuestro diseño está conformado por mujeres atravesadas por procesos migratorios de la ciudad de Ushuaia, que han accedido a la formación docente.

Respecto del proceso de análisis, se abordó como un continuo en el que "se intercalan períodos de campo con períodos de análisis y elaboración conceptual" (Rockwell, 2015a, p. 67). En él se fueron construyendo descripciones analíticas que explicitan las relaciones emergentes en un doble movimiento, la doble hermenéutica como el modo de construir conceptos a partir de la reinterpretación de situaciones ya significadas por los participantes. Se trata de lo que Geertz (2003) llamó una descripción densa: una particular integración de descripción y teoría característica de este enfoque.

A partir de la reconstrucción de las trayectorias escolares que se realizó a lo largo de esta investigación, se plantea que las experiencias vividas por estas mujeres en los procesos migratorios atravesados, en diálogo con la formación docente, favorecieron la construcción de nuevos sentidos respecto de sus condiciones materiales y simbólicas de vida, y entrever perspectivas para transformarlas.

\section{ALGUNOS ANTECEDENTES Y REFERENTES TEÓRICOS}

Abordar las desigualdades se encuentra lejos de tener una única perspectiva. En este trabajo dicho concepto no se ciñe solo a la pobreza, en tanto esta se puede entender como un subproducto de variadas inequidades, y la desigualdad una noción relacional, compleja y multidimensional (Kessler, 2014). Para este enfoque relacional (Tilly, 2000), las desigualdades son percibidas como el resultado de un conjunto de prácticas que las generan (Dubet, 2015). Si bien las desigualdades 
económicas atraviesan al resto, una mirada multidimensional del fenómeno insinúa pensar la interrelación entre las variadas dimensiones que atraviesan lo social, analizando causas y consecuencias en contextos determinados.

Si nos referimos a las desigualdades educativas, además de considerar las tradicionales variables socioeconómicas, también tendremos en cuenta las condiciones que las habilitan y las particularidades que contribuyen a su configuración. Ya que, a pesar del discurso histórico de la igualdad, el sistema público de educación argentino sostiene que, por medio de ciertas prácticas y representaciones, formas de desigualdad que reproducen las diferencias sociales que configuran sus propias huellas (Dussel, 2009).

Respecto del nivel educativo superior, Bourdieu y Passeron (1998) argumentan que la desigualdad social con la que los niños comienzan la escolaridad, no solo no es eliminada en sus trayectorias escolares, sino que es redoblada por el accionar de la institución escolar, reproduciendo o aumentando la desigualdad de origen. También en Argentina, como en otros países, en los últimos tiempos "la desigualdad se corre hacia arriba, hacia los niveles superiores de la escolaridad básica y post-básica" (Fitoussi y Savidan, 2003, citado en Tiramonti y Montes, 2009, p. 8).

Con la intención de dar cuenta de esta multiplicidad de dimensiones y procesos en juego en estos procesos, partimos de recuperar la noción de trayectorias escolares como los recorridos por las instituciones educativas en el marco de una densa trama que comprende un abanico heterogéneo de actores sociales e instituciones. Esta trama se va entretejiendo significativamente por medio de las experiencias, los sentidos y las prácticas de los sujetos integrando múltiples mediaciones. Como menciona Elena Achilli (2017), retomando la noción gramsciana de sentido común:

Los sujetos, como sujetos sociales, producen interpretaciones de sus prácticas y relaciones (...) son conocedores de lo que hacen $\mathrm{y}$, a la vez, poseedores de un sentido común cargado de una multiplicidad de huellas que desconoce. Un sentido común con variadas cristalizaciones 


\section{Julieta Taquini}

en su conciencia práctica, con incorporación de fragmentos del conocimiento científico, con múltiples creencias, con aspectos derivados de las concepciones hegemónicas o de otras concepciones embrionarias, alternativas, diferentes (p. 13).

En esta línea de pensamiento, retomamos el desafío de Thompson de recuperar el rol protagónico de los sujetos sociales en tanto nos permite conferir "una dimensión relevante a la actividad creadora de los sujetos como hacedores de la historia" (Sorgentini, 2000, p. 66). En principio, el concepto de experiencia según Thompson (1981) alude a un tipo de conocimiento social que media entre los sujetos y la conciencia social. Esta noción, recupera la racionalidad de los sujetos en diálogo con la estructura objetiva que los trasciende.

Entonces, interesa dar cuenta de las particularidades que adquieren las trayectorias escolares en condiciones de desigualdad social y educativa de mujeres atravesadas por procesos de migración; atendiendo a los hitos, dificultades y procesos que construyen las condiciones para el tránsito de la formación docente. Sostenemos como Kaplan (2006), que las dimensiones sociales y personales se interrelacionan mutuamente y configuran trayectorias escolares con características singulares, que, analizadas desde los sentidos que se les adjudican, a partir de relaciones intersubjetivas en determinadas condiciones materiales y socio históricas, dan cuenta de fenómenos socioeducativos más amplios (Achilli, 2005).

Por otra parte, la perspectiva de género e interseccional (Crenshaw, 1989) nos permite percibir e interpelar aquellas prácticas arbitrarias y manifestaciones y argumentos de corte patriarcal, que discriminan y excluyen a las mujeres a partir de los estereotipos y las supuestas esencias culturales que confunden lo biológico con lo socialmente construido (Rockwell, 2015b). Entendemos que esta mirada es necesaria ya que, en el campo educativo, como en cualquier otro campo de la sociedad, dichas prácticas se hacen presentes dado el carácter estructural y transversal del patriarcado (Femenías, 2008). Desde hace más de una década Graciela 
Morgade (2012), insiste en que la educación formal interviene en la construcción de sujetos y cuerpos según una división sexual del trabajo de características tradicionales. En el caso de las mujeres migrantes de esta investigación, la complejidad de estos procesos interpela los estereotipos y las supuestas esencias culturales y de género en donde se mezclan lo biológico con lo socialmente construido (Rockwell, 2015b) produciendo y reproduciendo relaciones de desigualdad.

En Argentina, los estudios interesados por la relación escuela y desigualdad se remontan a los trabajos de Cecilia Braslavsky (1985). Luego, a partir de la implementación de la Ley de Educación Nacional №26206 (Congreso de la República Argentina, 2006) la investigación educativa argentina se ha enfocado fuertemente en analizar los fenómenos en torno a los procesos de inclusión, especialmente en el nivel de educación secundaria dado que, en este período particular, se ha tornado obligatoria (Boticelli y Borzese, 2014; Krichesky et al., 2010).

Significativamente menor es la producción académica sobre el nivel superior de educación. Adriana Chiroleu (2013) plantea que, a pesar de que nuevos grupos sociales acceden al nivel, no se han reducido las desigualdades sociales ni educativas. En ese sentido Ezcurra (2011) señala que la deserción, el fracaso, la segmentación, y las diferencias de capital cultural del estudiantado, ponen en duda su inclusión y fortalecen la noción desarrollada por Gentili (2009) de "exclusión incluyente".

En cuanto a la participación de las mujeres en ese nivel educativo, aunque mayor en la actualidad, existen campos del conocimiento en donde su presencia continúa siendo menor que la de los varones (De Garay y Del Valle, 2011). En este sentido nos interesa observar que, en Argentina, la profesión docente es mayoritariamente femenina (DINIECE, 2004; Centro de Implementación de Políticas Públicas para la Equidad y el Crecimiento (CIPPEC), 2016; Secretaría de Evaluación Educativa, 2016). Y Poliak (2009), Charovsky (2013) y Birgin (2013) dan cuenta de que las trayectorias educativas desiguales inciden luego en la formación de quienes eligen esa profesión.

Por su parte, Caggiano (2019), Caggiano y Segura (2014), Courtis y Pacecca (2010) y Magliano (2007a, 2007b, 2009) sostienen que las INTEREDU № 5 Vol. II (DiCIEMBRE 2021) PÁGs. 41-67. ISSN: 2735-6523| 49 


\section{Julieta Taquini}

dimensiones de género, etnia y clase social deben analizarse conjuntamente, "puesto que se refuerzan recíprocamente, profundizando las prácticas y discursos de exclusión que ha enfrentado históricamente un amplio conjunto de las mujeres bolivianas en este país" (Magliano, 2009, p. 349). La intersección e imbricación de estas condiciones de desigualdad dan cuenta de trayectorias educativas particulares (Palacios et al., 2015). Por ello, se consideran los aportes de las investigaciones etnográficas realizadas por los equipos dirigidos por Neufeld y Thisted (2005) sobre los procesos socioculturales en las escuelas en contextos interculturales caracterizados por las desigualdades, como así también, aquellos estudios que prestan especial atención a las relaciones entre los sujetos y los contextos en las diferentes escalas a partir de la vida cotidiana en las escuelas: Rockwell (1995), Ezpeleta y Rockwell (1985), Achilli (2003, 2009), Santillán (2007) y Diez (2015). Por su parte, los trabajos de Ossola (2014, 2016a, 2016b, 2018) aportan una mirada específica sobre la educación intercultural en el nivel superior y la cuestión del lenguaje, en los procesos de formación desde una perspectiva etnográfica.

Por lo expuesto, y dado que al presente no existe conocimiento local al respecto, se espera que este trabajo contribuya a la reflexión sobre las condiciones de acceso, permanencia y finalización de las mujeres de origen boliviano en la formación docente de la ciudad de Ushuaia. Como así también, inscribirlo en una discusión más amplia y poder realizar un aporte sobre la relación entre los procesos migratorios y las condiciones de desigualdad en el nivel superior de educación.

\section{BREVE APROXIMACIÓN TEÓRICO METODOLÓGICA}

Tal como se presentó en la introducción, esta investigación fue realizada desde un enfoque socioantropológico que recupera la tradición etnográfica de la antropología desde una perspectiva crítica (Achilli, 2003, 2005, 2009, 2015, 2017). Esta decisión habilitó un diálogo interdisciplinario que permitió superar fragmentaciones y reducciones ahistóricas haciendo un "esfuerzo por relacionar las distintas dimensiones de una problemática 50 | INTEREDU № 5 Vol. II (DiCIEMBRe 2021) PÁGS. 41-67. ISSN: 2735-6523 
analizando los procesos que se generan en sus interdependencias y relaciones históricas contextuales" (Achilli, 2005, p. 17). Este modo de entender el proceso de producción de conocimiento llevó a tener especial interés por la cotidianeidad social, la recuperación del protagonismo de los sujetos sociales y la dialéctica entre trabajo de campo y trabajo conceptual.

El desafío de este enfoque consiste en hacer posible la inteligibilidad de la cotidianeidad social al contextualizar y relacionar las diferentes escalas e identificar las conexiones profundas "que las transformaciones políticas, económicas, culturales van anclando en las prácticas, significaciones y procesos que diariamente construyen los sujetos" (Achilli, 2005, p. 107).

Esta forma de proceder en la investigación se presenta coherente respecto del enfoque propuesto, ya que la etnografía desde este enfoque crítico - al integrar método y teoría, así como la diversidad de actores, sus prácticas y construcciones de sentido- permitió poner en relación las biografías particulares con la historia social e institucional que les toca vivir a los sujetos.

En cuanto al trabajo de campo, este se sitúa en Ushuaia entre los años 2016 y 2019, y fue realizado centralmente con seis mujeres que residían en la ciudad y que habían estado atravesadas de alguna manera por procesos migratorios con origen en Bolivia. Al respecto cabe aclarar que las mujeres fueron informadas del objetivo de la investigación, y su participación como entrevistadas, contó con el consentimiento oral informado y registrado en soporte de audio.

Tal como indicamos previamente, las principales estrategias de construcción de la información que utilizamos fueron entrevistas en profundidad a las mujeres de la investigación, cuyo resultado pudo triangularse con el análisis de fuentes documentales, observaciones y entrevistas a otras personas vinculadas a ellas.

Las entrevistas se realizaron - recuperando las recomendaciones de Achilli (2005) - , en varias instancias y principalmente en dos tiempos. En los primeros encuentros, mediante preguntas de corte descriptivo, solicitamos a las mujeres que desarrollen los temas de manera abierta y INTEREDU № 5 Vol. II (DiCIEMBRE 2021) PÁGs. 41-67. ISSN: 2735-6523| 51 


\section{Julieta Taquini}

sin direccionalidad. Así fue que conversamos sobre sus familias, el barrio, la ciudad y la escuela en sus lugares de origen. Luego nos adentramos en el proceso migratorio, la vida cotidiana ya en Ushuaia, cómo fue estudiar en esa ciudad y, particularmente, sobre la experiencia en el nivel superior de educación. Estas interrogantes guiaron los primeros encuentros con el objetivo de ayudarnos a "descubrir preguntas" para los posteriores. Luego, la siguiente etapa de entrevistas contribuyó para ir abriendo la conversación, ya más dirigidamente, con el fin de ampliar y profundizar en las categorías significadas en la primera instancia.

Si bien el vínculo más frecuente e intenso fue con las mujeres de la investigación, no por ello menos significativos fueron los encuentros informales con docentes, familiares y amistades de ellas. Es decir, que otras personas también proporcionaron nuevas perspectivas a la temática en cuestión a modo "muestreo en cadena o bola de nieve" (Hernández Sampieri et al., 2006). Pudimos conversar con algunos profesores de las mujeres entrevistadas y con dos tutoras del Departamento de Orientación al Estudiante de uno de los Institutos de Formación Docente (IFD), con el padre y la madre de una de ellas, y con algunas amistades también.

El encuentro con estas personas allegadas a las mujeres tuvo que ver con la necesidad de "estar allí" (Geertz, 1997), compartir distintos espacios y momentos que nos permitieran acercarnos y construir una relación con estas mujeres a quienes finalmente les propondríamos la realización de entrevistas.

Con algunas de las ellas compartimos reuniones y eventos familiares (celebraciones de cumpleaños, bautismos, entre otros menos formales como mates, asados, entre otros) en las que pudimos conocerlas, ya que tenemos amistades en común. En otras ocasiones, las frecuentábamos dada nuestra condición de docente en el IFD en el que varias de las mujeres estudiaban al momento de llevar adelante el trabajo de campo. De ahí que compartir un mismo espacio institucional permitió un acercamiento que se fue dando en el tiempo, y lo suficientemente cotidiano para poder establecer un tipo de relación con suficiente confianza.

52 | INTEREDU № 5 VOL. II (DicIEMBRE 2021) PÁGS. 41-67. ISSN: 2735-6523 
Las mujeres habían accedido a la formación docente en diferentes institutos de gestión estatal, aunque en distintos momentos y lugares. Aunque no todas ellas nacieron en Bolivia, sus trayectorias escolares se encuentran entramadas en procesos migratorios protagonizados por padres, madres y abuelos produciendo condiciones y experiencias particulares. Al respecto se hace necesario considerar que la categoría de "boliviano" es usada corrientemente en Argentina para incluir a quienes nacieron en ese país y a sus descendientes (Grimson, 2006). También Mallimaci (2011) afirma que las generaciones posteriores a quienes han migrado de Bolivia, es decir, a argentinos descendientes de bolivianos, son referidos en la cotidianeidad como bolivianos. Atendiendo a lo especificado, entrevistamos a tres generaciones diferentes de mujeres de la ciudad de Ushuaia que se reconocen "de origen boliviano": nacidas en Bolivia, hijas de bolivianos y nietas de bolivianos.

Rosa ${ }^{5}$ tenía 32 años cuando la entrevistamos. Nació en Cochabamba, Bolivia, y migró a Ushuaia a fines del 2000. Finalizó sus estudios secundarios en esa ciudad en un centro educativo para adultos. Al momento de ser entrevistada vivía con sus dos hijas y su pareja, se encontraba cursando el último año de la carrera de formación docente en un IFD público de gestión estatal de la provincia de Tierra del Fuego; y trabajaba como preceptora en una escuela de la ciudad.

Yanina tenía 20 años al momento de entrevistarla. Ella también nació en Bolivia (Punata). Sus padres la llevaron a vivir a Ushuaia cuando tenía dos años de edad aproximadamente. Después de intentar otros caminos de desarrollo profesional y laboral, ingresó a un IFD de la ciudad, y al momento de las entrevistas, no trabajaba y se hallaba cursando el primer año del Profesorado de Educación Inicial.

Micaela nació en Ushuaia, pero es hija de inmigrantes bolivianos. Realizó y finalizó todos los niveles de educación obligatoria en instituciones educativas de gestión estatal de esa ciudad. Al momento de entrevistarla tenía 22 años y se encontraba cursando el tercer año del

\footnotetext{
${ }^{5}$ Los nombres que aparecen fueron modificados con el fin de preservar la identidad de las entrevistadas.
}

INTEREDU № 5 VOL. II (Diciembre 2021) PÁGs. 41-67. ISSN: 2735-6523| 53 


\section{Julieta Taquini}

Profesorado de Inglés en un IFD público de gestión estatal. No trabajaba formalmente, y vivía con su familia: padre, madre, hermanos, cuñados, y sobrinos, a quienes cuidaba cuando sus padres o madres trabajaban fuera de la casa. Además, daba clases particulares de inglés los sábados, en la Iglesia a la que asistía.

María es la mayor de las entrevistadas. Nació en 1979 en la provincia de Salta, y su padre y su madre bolivianos migraron a Argentina durante aquella década. Vivía con su marido y su hija, y trabajaba en una escuela primaria de la ciudad de Ushuaia. Su escolaridad atravesó la ruralidad, la educación común y la técnica superior hasta que, finalmente una vez en Ushuaia, finalizó el Profesorado de Educación Primaria en uno de los dos IFD de la ciudad.

Olga es nieta de bolivianos inmigrantes de Jujuy, provincia en la que nació. Partió a Ushuaia un año después de finalizar la escuela secundaria, cuando tenía 18 años. Cuando la entrevistamos estaba casi finalizando el Profesorado de Lengua y Literatura. Se conformó como referente de la comisión de género en el IFD donde estudiaba, y además, participaba activamente en organizaciones de derechos humanos y pueblos originarios. Al poco tiempo se recibió de profesora, y actualmente continúa estudiando el Profesorado de Historia, en el mismo Instituto.

Violeta nació en un pequeño pueblo de Jujuy limítrofe con Bolivia. $\mathrm{Su}$ padre es salteño, y su madre, jujeña. Desconoce el origen de sus abuelos, sin embargo, se reconoce como miembro de una comunidad con la que comparte tradiciones, historia y prácticas con comunidades de Bolivia. Estudió el Profesorado de Educación Primaria en su provincia natal y migró a Ushuaia hace dos años cuando se recibió de maestra. En la actualidad se desempeña como docente suplente en dos escuelas primarias, y cursa la licenciatura en Gestión Educativa en la Universidad Nacional de Tierra del Fuego. Tenía 22 años cuando nos conocimos.

En cuanto al proceso de análisis, dado el carácter flexible de nuestro diseño, fue simultáneo al trabajo de campo: un proceso recursivo en el que las construcciones hipotéticas van relacionando de manera recursiva 54 | INTEREDU № 5 VOL. II (DiCIEMBRE 2021) PÁGS. 41-67. ISSN: 2735-6523 
el material concreto del trabajo de campo con las categorías teóricas seleccionadas para ello (Achilli, 2009). Se trató de un proceso continuo en el que alternamos los períodos del trabajo de campo con los de análisis de elaboración conceptual. En este continuo se fueron construyendo descripciones analíticas que explicitan las relaciones emergentes en un doble movimiento de reinterpretación de situaciones ya significadas por las participantes.

En esta investigación la descripción de los cruces entre los condicionamientos estructurales (acceso al mercado de trabajo, ingresos, género, origen, edad, entre otros), los procesos cotidianos, las prácticas y los sentidos de los sujetos, se realizó mediante un proceso de análisis que pretendió relacionar fragmentos de informaciones diversas. A partir de lo que las personas entrevistadas expresaron, pudimos identificar conflictos, mediaciones y resistencias, como así también tendencias y continuidades desde cierta direccionalidad teórica (Achilli, 2005). Luego, el producto del trabajo analítico resultó en un texto en el que se intentó “documentar lo no documentado" (Rockwell, 2015a) de las formas variadas y particulares de las trayectorias de las mujeres de manera descriptiva.

LA FORMACIÓN DOCENTE Y LA RESIGNIFICACIÓN DE SENTIDOS: ALGUNOS HALLAZGOS

A partir del análisis realizado podemos sostener que, en los procesos de construcción de las trayectorias escolares, las experiencias formativas de estas mujeres - en el cruce con otras experiencias y procesos - devienen en nuevas significaciones, que presentan tanto continuidades como especificidades. Es destacado por las entrevistadas que el paso por la formación docente favoreció la apropiación de marcos conceptuales de interpretación que las han habilitado a resignificar distintos aspectos de sus trayectorias escolares y experiencias de vida habilitando, simultáneamente, la transformación de la propia práctica docente.

Con esto queremos decir que la autopercepción y la valoración positiva respecto de su propia condición de mujer inmigrante de origen boliviano habilitaron el desarrollo de un conjunto de habilidades, saberes 


\section{Julieta Taquini}

y estrategias que les permitieron participar activamente en el proceso de construcción de sentidos que circulan socialmente, y de los cuales emergen los discursos y las prácticas de discriminación hacia la comunidad a la que autoadscriben.

Entre ellos, destacamos los sentidos construidos sobre:

- Las condiciones materiales y simbólicas de sus trayectorias escolares.

La toma de conciencia por parte de las mujeres sobre las condiciones materiales y simbólicas en que han desarrollado sus trayectorias escolares resulta en una reflexión sobre cómo estas incidieron o inciden en sus propios procesos escolares. En palabras de una de las entrevistadas, la formación docente le implicó un proceso relacionado con una dimensión identitaria que antes no había sido vivida:

¿Tal vez más como identidad? Creo que ha sido lo que más me ha llegado en todo este tiempo, por esto de mis papás. O sea, de yo haber estado avergonzada tal vez de, capaz, el hecho de que mi papá es albañil, que mi mamá trabajó todo el tiempo en casa, el hecho de que me llevaba a la escuela 31 pero a la noche mi mamá "iba" a la escuela 31 (...) Yo creo que en ese tiempo puedo ver de dónde vengo, y no tener vergüenza (Micaela, comunicación personal, 7 de mayo de 2019).

- El rol de la lengua materna en las experiencias escolares y en los procesos de construcción de identidad colectiva.

En los relatos de las mujeres de la investigación se puso de manifiesto que la formación docente les proporcionó experiencias formativas que favorecieron la reflexión sobre los procesos de discriminación racial y de desigualdad. Simultáneamente, diremos que estas experiencias articuladas, promovieron otros procesos de reflexión 56 | INTEREDU № 5 VOL. II (DiCIEMBRE 2021) PÁGs. 41-67. ISSN: 2735-6523 
vinculados a la diversidad étnica y la equidad. Al respecto recuerda una de las mujeres:

Yo igual, que me rebelé con el quechua porque no quería...llamar la atención...como que cuando vine acá descubrí mis raíces. Ahí si es otra cosa ya (Rosa, comunicación personal, 4 de noviembre de 2017).

- Sus propias trayectorias escolares en clave de género.

En estas historias, la formación docente emerge en estas trayectorias escolares como un recurso concreto para cuestionar y dar visibilidad a las situaciones de subalternidad y desigualdad de género, e impulsar transgresiones a las reglas de la sociedad patriarcal resultando en la toma de conciencia de esas desigualdades.

En el IPES me di cuenta que siempre había sido feminista, - nos dijo Olga- (comunicación personal, 13 de julio de 2018).

Pensamos que estas mujeres lograron sortear obstáculos y encarar los desafíos que la vida les propuso solo por el hecho de ser mujeres, con una actitud decidida y perseverante que, con el tiempo y en las experiencias en el nivel superior de educación, resultó en la apropiación de una perspectiva de género.

- La práctica docente y la escuela.

Sostenemos que los sentidos producidos en los procesos de formación docente, en diálogo con las condiciones en las que atravesaron la escolaridad las mujeres de las trayectorias escolares analizadas, les permitieron cuestionar ciertas prácticas y pensar otras que habilitan la transformación de las condiciones socioculturales en las que se desarrollan las trayectorias escolares de las futuras generaciones. De este modo, algunas prácticas de los sujetos que participan de la construcción 


\section{Julieta Taquini}

social de la escuela pueden favorecer procesos de reflexividad, crítica y hasta de transformación de los sentidos hegemónicos.

Violeta nos contó que en la Licenciatura en Gestión Educativa que actualmente cursa se estaba dando cuenta de,

que las lecturas, las charlas, las vivencias, [me] hicieron revalorizar, que gracias a la maestra y al maestro pude ser quien soy ahora y haber aprendido a leer y escribir... porque todos mis hermanos, hoy, ninguno estudió, y mi hermana mayor me cuidaba y ahora ella trabaja en el campo (...) hay una nena que nos dimos cuenta que no iba a la escuela, pero era que la habian traído de Bolivia, y cuidaba a sus hermanitos, estaba directamente de niñera, sin acceder al derecho a la educación como yo...para ellos es así, algunos estudian, algunos van a la escuela y algunos cuidan a sus hermanos, y después se trabaja en el campo: eso soy yo. De ahí vengo yo, es lo que yo viví (...) y cuando uno lo pasa...pasa por esas experiencias, uno está más alerta. Ahora en la escuela cuando digo Bolivia y alguien se ríe, paro y pregunto. Ahora puedo intervenir (Violeta, comunicación personal, 22 de noviembre de 2019).

Por tanto, podemos observar que, quienes en apariencia ya estaban signadas por las condiciones de desigualdad de sus trayectorias escolares, han transformado sus demandas y sus representaciones respecto de quiénes son, quiénes quieren ser y qué han querido y quieren hacer en la escuela.

\section{CONCLUSIONES Y NUEVOS INTERROGANTES}

El objetivo de este estudio fue realizar la descripción de las trayectorias escolares de mujeres de Ushuaia de origen boliviano atravesadas por procesos migratorios, a partir del análisis relacional entre dichos procesos y sus experiencias formativas. Es decir, se intentó dar cuenta de cómo son y cómo se configuran las trayectorias escolares a partir de los sentidos sobre la educación formal construidos en las experiencias formativas y, particularmente, en la formación docente. 
El aporte fundamental de este trabajo radica en que, desde el enfoque en el que nos posicionamos el protagonismo de las personas es central, y que por tanto no existen trayectorias prediseñadas ni definidas: los recorridos se hacen en el transcurso y articulación de diversos procesos, otorgándoles especificidades y particularidades a cada una de ellas.

Ciertamente, los procesos de escolarización habilitan a los sujetos a atribuir sentidos a la educación formal, que resultan en la posibilidad de promover y producir estrategias de acceso, sostenimiento y finalización de los diferentes niveles educativos. En este sentido, entendimos que si bien los sujetos en sus prácticas recuperan y se apropian de los recursos culturales disponibles en los contextos en los que desarrollan sus trayectorias educativas, respecto de las condiciones objetivas en las que se encuentran, el mundo social es una construcción dinámica y contradictoria (Achilli, 2003), en el que las personas tienen la posibilidad de modificar y alterar -en interacción con otros y bajo diversas condiciones sociohistóricas- los sentidos iniciales de esas prácticas en un proceso de resignificación a través del cual, recuperan o desechan dichos sentidos activamente (Santillán, 2007).

Hasta aquí, sin desconocer las limitaciones existentes, podemos decir con Courtis y Pacecca (2010) que:

independientemente de la escasa o nula modificación de las estructuras de subordinación, los sujetos que atraviesan la experiencia migratoria suelen modificar su propia percepción de sí mismas, en particular en relación a su agencia y a su capacidad de gestionar situaciones complejas y de incertidumbre. Así, entre los puntos pendientes de análisis podemos señalar las potencialidades emancipadoras que la migración puede acarrear para las mujeres, más allá de su percepción como experiencia positiva en casos individuales (p.18).

El análisis de las entrevistas y del trabajo de campo en su totalidad nos permitió visibilizar que los sentidos construidos en la formación docente sobre cómo se ven a sí mismas adquieren rasgos distintivos y singulares resultado de identificar y hacer consciente y visible sus 


\section{Julieta Taquini}

experiencias escolares. Sentidos que las mujeres han ido construyendo en sus trayectorias escolares y en el cruce con otras experiencias vividas.

Al desnaturalizar las condiciones y procesos imbricados en sus trayectorias, estas mujeres han podido resignificar sus propias experiencias escolares. Como resultado de ello, han podido reconocerse miembros de un colectivo que las excede pero que también las constituye como sujetos diferentes a los demás, y en tal sentido, han sabido reconocer la posibilidad de propiciar, mantener, modificar o transformar las relaciones sociales que se desarrollan en los procesos educativos.

Por tanto, aquellos sentidos atribuidos a la educación formal -la escolarización en cualquiera de sus niveles - a partir de la formación docente han sido de importancia decisiva a la hora de generar y crear estrategias de sostenimiento y finalización de dicho nivel educativo.

Por lo pronto, en el análisis realizado hasta aquí, el foco estuvo puesto en recuperar las dimensiones que configuran las trayectorias escolares de mujeres atravesadas por procesos migratorios que ingresan a la formación docente, con el acento colocado en los sentidos, prácticas y estrategias que ellas mismas construyeron en esos recorridos. Continúa el desafío de dar cuenta de otros aspectos de un sistema formador en contextos migratorios en clave insular, que recupere las características y las posibilidades que ello significa.

\section{REFERÊNCIAS BIBLIOGRÁFICAS}

Achilli, E. (2003). Escuela, familia y etnicidades: Investigación socioantropológica en contextos interculturales de pobreza urbana [Tesis de doctorado, Universidad de Buenos Aires]. Repositorio institucional. http://repositorio.filo.uba.ar/handle/filodigital/4095

Achilli, E. (2005). Investigar en Antropología Social. Los desafíos de transmitir un oficio. Laborde Libros Editor.

Achilli, E. (2009). Escuela, familia y desigualdad social. Una antropología en tiempos neoliberales. Laborde Libros Editor.

60 | INTEREDU № 5 VOL. II (DiCIEMBRE 2021) PÁGS. 41-67. ISSN: 2735-6523 
Achilli, E. (2015). Hacer antropología. Los desafíos del análisis a distintas escalas. Boletín de Antropología y Educación, 6(9), 103-107. http://antropologia.institutos.filo.uba.ar/sites/antropologia.institut os.filo.uba.ar/files/bae_n09_e02a01.pdf

Achilli, E. (2017). Construcción de conocimientos antropológicos y coinvestigación etnográfica. Problemas y desafíos. Cuadernos de Antropología Social, 45, 7-20. https://doi.org/10.34096/cas.i45.3795

Birgin, A. (2013). Informe Argentina. Estudio sobre criterios de calidad y mejora de la formación docente en el Mercosur. Programa de Apoyo al Sector Educativo del Mercosur (Pasem). Universidad Nacional de San Martín. https://panorama.oei.org.ar/_dev2/wpcontent/uploads/2017/07/Relevamiento-de-los-criterios-de-calidady-mejora.pdf

Boticelli, L. y Borzese, D. (2014). Inclusión con calidad educativa para todos los jóvenes. Algunos datos para la reflexión y la acción en $\mathrm{M}$. Krichesky (Comp.), Adolescentes e inclusión educativa. Un derecho en cuestión (pp. 113-135). Noveduc Libros.

Bourdieu, P. y Passeron, J. (1998). La reproducción: elementos para una teoría del sistema de enseñanza. Fontamara.

Braslavsky, C. (1985). La discriminación educativa en la Argentina. GEL FLACSO.

Caggiano, S. (2019). Las migraciones como campo de batallas. Desigualdades, pertenencias y conflicto en torno a la movilidad de las personas. Dávila y Miño.

Caggiano, S. y Segura, R. (2014). Migración, fronteras y desplazamientos en la ciudad. Dinámicas de la alteridad urbana en Buenos Aires. Revista de Estudios Sociales, 48, 29-42. http://dx.doi.org/10.7440/res48.2014.03

Centro de Implementación de Políticas Públicas para la Equidad y el Crecimiento (CIPPEC). (2016). Informe Características y voces de los docentes. Serie de informes temáticos/2. Programa de Educación de la Secretaría de Evaluación Educativa del Ministerio de Educación de la Nación. 


\section{Julieta Taquini}

https://www.argentina.gob.ar/sites/default/files/caracteristicas_y_ voces_de_los_docentes_web_a4_simple.pdf

Charovsky, M.M. (2013). La fragmentación de la formación docente. Su relación con el sistema educativo. Estudio de caso en el partido de Pilar, provincia de Buenos Aires. [Tesis de Maestría, Universidad de Buenos Aires]. Repositorio institucional. http://repositorio.filo.uba.ar/handle/filodigital/4270

Chiroleu, A. (2013). Políticas públicas de Educación Superior en América Latina: ¿democratización o expansión de las oportunidades en el nivel superior? Espacio Abierto, 22(2), 279-304. https://www.redalyc.org/comocitar.oa?id=12226914006

Courtis, C. y Pacecca, M. I. (2010). Género y trayectoria migratoria: mujeres migrantes y trabajo doméstico en el Área Metropolitana de Buenos Aires. Papeles de Población, 16(63), 155-185. http://www.scielo.org.mx/scielo.php?script=sci_arttext\&pid=S1405 $-74252010000100006$

Congreso de la República Argentina (1995, 10 de agosto). Ley 24521. Por la cual se expide la Ley de Educación Superior. Boletín Oficial de la República Argentina (BORA).

Congreso de la República Argentina (2006, 28 de diciembre). Ley 26206. Por la cual se expide la Ley de Educación Nacional. Boletín Oficial de la República Argentina (BORA).

Congreso de la República Argentina (2010, 6 de mayo). Ley 25871. Por la cual se expide la Ley de Migraciones. Boletín Oficial de la República Argentina (BORA).

Crenshaw, K. (1989). Demarginalizing the Intersection of Race and Sex: A Black Feminist Critique of Antidiscrimination Doctrine, Feminist Theory and Antiracist Politics. University of Chicago Legal Forum, 1989(8), 139-167.

https://chicagounbound.uchicago.edu/uclf/vol1989/iss1/8/

De Garay, A. y Del Valle, G. (2011). Una mirada a la presencia de las mujeres en la educación superior en México. Revista 
Iberoamericana de Educación Superior, 3(6), 3 -30. http://www.scielo.org.mx/pdf/ries/v3n6/v3n6a1.pdf

Diez, M.L. (2015). Migración, biografías infantiles y procesos de identificación: Reflexiones desde una etnografía escolar en el sur de la ciudad de Buenos Aires. [Tesis de doctorado, Universidad de Buenos Aires]. Repositorio institucional. http://repositorio.filo.uba.ar/handle/filodigital/2952

Dirié, C. y Sosa, M. (2014). Alumnos extranjeros en el sistema educativo argentino: ¿cuántos son y dónde están? Población de Buenos Aires, 11(19), p. 31-47.

https://www.redalyc.org/articulo.oa?id=74030548002

Dirección Nacional de Información y Evaluación de la calidad educativa (DINIECE). Censo Nacional de Docentes 2004. Ministerio de Educación, Ciencia y Tecnología.

http://repositorio.educacion.gov.ar/dspace/handle/123456789/109857

Dirección Nacional de Información y Evaluación de la Calidad Educativa (DINIECE). (2010). Informe La Educación Argentina en Cifras.

Ministerio de Educación.

http://www.bnm.me.gov.ar/giga1/documentos/EL005565.pdf

Dubet, F. (2015). ¿Por qué preferimos la desigualdad? (aunque digamos lo contrario). Siglo XXI editores.

Dussel, I. (2009). ¿Qué lugar tiene la escuela media en la producción y reproducción de la desigualdad? Elementos para el debate. Revista de política educativa: revista de investigación de la Escuela de Educación Buenos Aires, 1, 68-90. http://hdl.handle.net/10908/881

Ezcurra, A. M. (2011). La igualdad como punto de partida en Igualdad en Educación Superior: un desafío mundial. Revista Argentina de Educación Superior.

http://www.untref.edu.ar/raes/documentos/raes_4_ostrovieski.pdf

Ezpeleta, J. y Rockwell, E. (1985). La escuela: relato de un proceso de construcción teórica. Revista Colombiana de Educación, 12. https://doi.org/10.17227/01203916.5093

Femenías, L. (2008). Violencia contra las mujeres: urdimbres que marcan la trama. En L. Femenías y E. Aponte Sánchez (Comps.), INTEREDU № 5 Vol. II (DiCIEMBRE 2021) PÁGs. 41-67. ISSN: 2735-6523| 63 


\section{Julieta Taquini}

Articulaciones sobre la violencia contra las mujeres (pp. 13-53). Editorial de la Universidad de la Plata. http://sedici.unlp.edu.ar/handle/10915/35346

Geertz, C. (1997). Estar allí. La antropología y la escena de la escritura. En El antropólogo como autor (11-34). Paidós.

Geertz, C. (2003). La interpretación de las culturas (12va edición). Gedisa. Gentili, P. (2009). Marchas y contramarchas. El derecho a la educación y las dinámicas de exclusión incluyente en América Latina (A sesenta años de la declaración universal de los derechos humanos). Revista iberoamericana de educación, 49, 19-57. https://rieoei.org/historico/documentos/rie49a01.pdf

Grimson, A. (2006). Nuevas xenofobias, nuevas políticas étnicas en Argentina. En A. Grimson y E. Jelin (Comps.), Migraciones regionales hacia la Argentina. Diferencias, desigualdades y derechos (pp. 69-97). Prometeo.

Hecht, A. C. y Schmidt, M. (2016). Introducción. La importancia del rol docente en las políticas interculturales en educación. En C. Hecht y M. A. Schmidt (Comps.), Maestros de la Educación Intercultural Bilingüe. Regulaciones, experiencias y desafíos (pp. 9 - 25). Noveduc.

Hermida, M. y Van Aert, P. (2013). Migración en Tierra del Fuego (o la historia de una ida y una vuelta). Sociedad Fueguina, 2, 5-12. http://www.untdf.edu.ar/uploads/archivos/02_Sociedad_Fueguina _Nro_2_1447941417.pdf

Hernández Sampieri, R., Fernández Collado, C., y Baptista Lucio, P. (2006). Metodología de la Investigación (5ta Edición). Editorial Mc Graw Hill.

Instituto Nacional de Estadísticas y Censos (INDEC). Censo Nacional de Población, Hogares y Viviendas 2001 y 2010. http://www.indec.gov.ar 
Kaplan, C. (2006). Trayectorias estudiantiles: las implicancias del oficio de alumno. En La inclusión como posibilidad (p. 38-43) Buenos Aires: Ministerio de Educación, Ciencia y Tecnología de la Nación.

Kessler, G. (2014). Controversias sobre la desigualdad. Argentina, 20032013. Fondo de Cultura Económica.

Krichesky, G., Cortelezzi, M., Cura, D. y Morrone, A. (2010). Jóvenes que miran a la escuela: Una investigación acerca de los procesos de inclusión/exclusión en la escuela secundaria. Fundación Cimientos.

Mallimaci Barral, A. I. (2010). Construyendo comunidades. Géneros, tiempos, espacios y memorias de los/as bolivianos/as en Ushuaia [Tesis de doctorado no publicada]. Universidad de Buenos Aires.

Mallimaci Barral, A. I. (2011). Las lógicas de la discriminación. Nuevo Mundo Mundos Nuevos.

https://doi.org/10.4000/nuevomundo.60921

Magliano, M. J. (2007a). Migración de mujeres bolivianas hacia Argentina: cambios y continuidades en las relaciones de género. Diagnóstico y propuestas. Amérique Latine Histoire et Mémoire. Les Cahiers ALHIM. 14. https://doi.org/10.4000/alhim.2102

Magliano, M. J. (2007b). Migración y género: La migración de mujeres bolivianas hacia Argentina en la segunda mitad del siglo XX. Cuadernos del Sur, Historia, 35-36, 441-467. http://bibliotecadigital.uns.edu.ar/scielo.php?script=sci_arttext\&pi $\mathrm{d}=$ S1668-76042007001100116\&lng=pt\&nrm=iso

Magliano, M. J. (2009). Migración, género y desigualdad social: la migración de mujeres bolivianas hacia Argentina. Estudios Feministas, 17, 349-367. http://dx.doi.org/10.1590/S0104-026X2009000200004

Martínez, L., Diez, M.L., Novaro, G. y Groisman, L. (2011). Migración e Interculturalidad: perspectivas de derechos y política educativa. Boletín de Antropología y Educación, 6(9), 57-62. http://antropologia.institutos.filo.uba.ar/sites/antropologia.institut os.filo.uba.ar/files/bae_n09_e01a09.pdf

Morgade, G. (2012). Aprender a ser mujer, aprender a ser varón. Relaciones de género y educación. Esbozo de un programa de acción. Noveduc. 


\section{Julieta Taquini}

Neufeld, A. y Thisted, J. (Comps.) (2005). De eso no se habla...los usos de la diversidad sociocultural en la escuela. EUDEBA.

Ossola, M. (2014). Diversidad cultural y lingüística en la educación superior Argentina. Debates sobre los usos y representaciones de las lenguas wichí y español entre jóvenes universitarios. Papeles de Trabajo, 27, 128-141. https://ri.conicet.gov.ar/handle/11336/6869

Ossola, M. (2016a). EIB y lengua quechua en Santiago del Estero. En C. Hecht y M. A. Schmidt (Comps.), Maestros de la Educación Intercultural Bilingüe. Regulaciones, experiencias y desafíos (pp. 123 - 140). Noveduc.

Ossola, M. (2016b). Pueblos indígenas y educación superior en la Argentina: debates emergentes. Revista del Centro de Investigaciones Sociales y Educativas del Norte Argentino CISEN, 4(1), 57-77. http://ppct.caicyt.gov.ar/index.php/cisen/article/view/8732

Ossola, M. (2018). Educación Superior y diversidad cultural. Análisis de las experiencias formativas de jóvenes indígenas en proyectos de extensión universitaria. Praxis Educativa, 22(3), 56-63. https://doi.org/10.19137/praxiseducativa-2018-220306

Palacios, M., Padawer, A., Hecht A. y Novaro G. (2015). Mujeres indígenas. Trayectorias educativas de tres referentes comunitarias en Argentina en G. Novaro, A. Padawer y A. C. Hecht (Coords.). En Educación, pueblos indígenas y migrantes. Reflexiones desde México, Bolivia, Argentina y España (pp. 161 - 190). Biblos.

Poliak, N. (2009). Fragmentación educativa en el campo docente: acerca de criterios de selección de profesores en escuelas medias de la ciudad de Buenos Aires. Espacios en Blanco. Revista de educación, 19, 267-298. http://www.redalyc.org/articulo.oa?id=384539801013

Rockwell, E. (1995). De huellas, bardas y veredas: una historia cotidiana en la escuela. En E. Rockwell, (Coord.), La escuela cotidiana (pp. 1357). Fondo de Cultura Económica.

Rockwell, E. (2015a). La experiencia etnográfica. Historia y cultura en los procesos educativos. Paidós.

66 | INTEREDU № 5 VOL. II (DicIEMBRE 2021) PÁGS. 41-67. ISSN: 2735-6523 
Rockwell, E. (2015b). Prólogo. En G. Novaro, A. Padawer y A. C. Hecht (Coords.), Educación, pueblos indígenas y migrantes. Reflexiones desde México, Bolivia, Argentina y España (pp. 11-39). Biblos.

Santillán, L. (2007). Trayectorias educativas y cotidianeidad. Una etnografía del problema de la educación y la experiencia escolar en contextos de desigualdad. [Tesis de doctorado, Universidad de Buenos Aires]. Repositorio institucional. http://repositorio.filo.uba.ar/handle/filodigital/4676

Secretaría de Evaluación Educativa (2016). Ministerio de Educación y Deportes. Informe de resultados Operativo Aprender 2016. http://www.bnm.me.gov.ar/giga1/documentos/EL005597.pdf

Sorgentini, H. (2000). La recuperación de la experiencia histórica: un comentario sobre E. P. Thompson. Sociohistórica, 7, 53-80. http://www.fuentesmemoria.fahce.unlp.edu.ar/art_revistas/pr.282 0/pr.2820.pdf

Taylor, S. J. y Bogdan, R. (1987). Introducción a los métodos cualitativos de investigación. Paidós.

Thompson, E. (1981). La miseria de la teoría. Editorial crítica. Tilly, Ch. (2000). La desigualdad persistente. Manantial.

Tiramonti, G. y Montes, N. (2009). La escuela media en debate: problemas actuales y perspectivas desde la investigación. Manantial. 